\title{
Assessing the Impact of Twin Pregnancies on the Pelvic Floor Using 3-Dimensional Sonography
}

\author{
A Pilot Study
}

\begin{abstract}
Juliana Sayuri Kubotani, MD, Edward Araujo Júnior, MD, PhD, Miriam Raquel Diniz Zanetti, PhD, Jurandir Piassi Passos, MD, Zsuzsanna Ilona Katalin de Jármy Di Bella, PhD, Julio Elito Júnior, PhD
\end{abstract}

Received August 12, 2013, from the Pelvic Floor Sector, Department of Obstetrics, Federal University of São Paulo, São Paulo, Brazil. Revision requested September 11, 2013. Revised manuscript accepted for publication November 4 , 2013.

Address correspondence to Edward Araujo Júnior, $M D, P h D$, Department of Obstetrics, Federal University of São Paulo, Rua Carlos Weber 956, Apartamento 113, Vila Leopoldina, 05303-000 São Paulo-SP, Brazil.

E-mail:araujojred@terra.com.br

Abbreviations

BMI, body mass index; 3D, 3-dimensional

doi:10.7863/ultra.33.7.1179
Objectives - The purpose of this study was to compare the morphologic characteristics of the pelvic floor musculature between women with twin and singleton pregnancies.

Methods-We conducted a cross-sectional case-control study of 40 nulliparous women aged 20 to 38 years to compare women with singleton pregnancies $(n=23)$ to women with twin pregnancies $(n=17)$. Biometric measurements of the levator hiatus and the sagittal and coronal diameters were made by transperineal 3-dimensional sonography between the 28th and 38th gestational weeks. Comparisons were statistically assessed by the unpaired Student $t$ test and Mann-Whitney $U$ test.

Results - For the women with singleton pregnancies, the mean sagittal diameters at rest, during the Valsalva maneuver, and during pelvic floor contraction were 5.3, 5.7, and $4.5 \mathrm{~cm}$, respectively, and the mean coronal diameters under these conditions were $3.8,4.1$, and $3.6 \mathrm{~cm}$. For the women with twin pregnancies, the corresponding values were as follows: mean sagittal diameters, $5.3,5.8$, and $4.6 \mathrm{~cm}$; and mean coronal diameters, $4.3,4.3$, and $3.8 \mathrm{~cm}$. The differences in coronal diameters were statistically significant at rest $(P<.01)$ and during contraction $(P=.04)$. The mean levator hiatal areas for the women with singleton pregnancies were $14.6,16.9$, and $11.7 \mathrm{~cm}^{2}$ at rest, during Valsalva, and during contraction, respectively; for the women with twin pregnancies, these values were $16.0,18.6$, and $12.6 \mathrm{~cm}^{2}$.

Conclusions - Hiatal measurements were higher in twin than in singleton pregnancies, with coronal diameters reaching significance at rest and during contraction, suggesting that pelvic support undergoes greater changes during twin pregnancy.

Key Words —obstetric ultrasound; pelvic floor; 3-dimensional sonography; twin pregnancy

$\mathrm{M}$ any physiologic alterations observed during pregnancy result from a combination of hormonal and mechanical factors that may cause changes to the pelvic floor ${ }^{1,2}$; these changes contribute to pelvic floor dysfunction irrespective of the type of delivery. ${ }^{3,4}$ The physical changes during twin pregnancy are more pronounced, with greater compression of the abdominal organs and higher elevation of the diaphragm than in singleton pregnancy. ${ }^{5}$ Nevertheless, few studies have evaluated how twin pregnancy affects the pelvic floor. Studies by Goldberg et al, ${ }^{6}$ Legendre et al, ${ }^{7}$ and Cuerva González et $\mathrm{al}^{8}$ revealed higher rates of stress urinary incontinence after twin deliveries; however, to our knowledge, no studies have directly evaluated the pelvic floor during pregnancy. 
This study aimed to assess the changes in pelvic floor support during twin pregnancy by comparing the musculature in twin and singleton pregnancies using transperineal 3-dimensional (3D) sonography during the third trimester of pregnancy.

\section{Materials and Methods}

\section{Design and Participants}

A cross-sectional case-control study comparing singleton and twin pregnancies was conducted between August 2011 and April 2013. Participants were randomly selected; they were nulliparous women, aged 20 to 38 years, and attending prenatal consultations at the outpatient clinics of the Department of Obstetrics, Federal University of São Paulo. Evaluations took place between the 28th and 38th gestational weeks. The study was approved by the Research Ethics Committee of the Federal University of São Paulo (number 0506/11), and all participants signed consent forms.

We excluded patients who had any of the following conditions: prepregnancy history of urinary incontinence, fecal incontinence, or genital prolapse; fetal abnormalities detected by sonography; prior intrauterine death at gestations later than 20 weeks; monochorionic twin pregnancies complicated by twin-twin transfusion, an acardiac fetus, or conjoined twins; and multiple pregnancies with 3 or more fetuses. The control group comprised nulliparous twin pregnant women with similar gestational ages. We did not control for maternal age, ethnic origin, or body mass index (BMI).

\section{Measurement Procedures}

Transperineal 3D sonography was performed with the patients in the lithotomy position after emptying their bladders. A convex volume transducer (covered with a sterile condom) with an automatic sweep (3-5 MHz) coupled to a Voluson E8 Expert ultrasound system (GE Healthcare, Zipf, Austria) was used. The labia minora were parted, and the transducer was positioned in the vaginal introitus and oriented in a medial sagittal plane with minimal pressure. We used an angle of $70^{\circ}$ or greater, which enabled viewing of the following structures from right to left: pubic symphysis, bladder neck, urethra, vagina, and distal rectum with the anorectal junction and proximal anal canal (Figure 1). All evaluations were performed by a single examiner (J.P.P.), who had 3 years of experience in transperineal 3D sonography.

The evaluations were performed under the following conditions: (1) at rest, (2) during the Valsalva maneuver, and (3) during pelvic floor contraction. Under each condition, measurements of the sagittal and coronal diameters and the levator hiatal area were made. At least 2 measurements were taken per condition per patient; the data were digitally stored before selecting the best quality images for final assessment. Data were transferred to a personal computer, and the evaluations were made at a later point with 4D View version 10.5 software (GE Healthcare), by another examiner (J.S.K.), who was blinded to the transperineal 3D sonographic examinations.

The sagittal diameter was identified as the minimum distance between the posterior hyperechoic part of the pubic symphysis and the anterior border of the pubovisceral muscle at the anorectal angle. The coronal diameter was measured at the widest part of the levator hiatus by tracing a line perpendicular to the sagittal diameter (Figure 2). The levator hiatus was defined as the area delineated by the pubovisceral muscle, pubic symphysis, and inferior pubic ramus, in the axial plane of the hiatal dimension (Figure 3), as described by Dietz et al. ${ }^{9}$

\section{Sample Size Calculation}

To determinate the number of participants needed for this study, we used Cochran's formula ${ }^{10}: \mathrm{n}=t^{2} \times \mathrm{P} \times(1-\mathrm{P}) / d^{2}$. Considering a normal distribution $P$, we can adopt $t=1.96$, with an area under the curve of 0.05 and an error margin of $5.0 \%$. $P$ was fixed at $1 \%(0.01)$; therefore, $(1-P)$ is 0.99 . Thus, we required of minimum of 16 participants per group.

\section{Statistical Analysis}

The study data were transferred to a spreadsheet in Excel 2007 (Microsoft Corporation, Redmond, WA) and were analyzed with SPSS version 15.0 software for Windows (IBM Corporation, Armonk, NY). The unpaired Student $t$ test and Mann-Whitney $U$ test were used to compare the data obtained from the transperineal 3D sonographic examinations between the groups. We used a statistical significance level of $P<.05$.

\section{Results}

We randomly enrolled 23 women with singleton pregnancies (mean age \pm SD, $29.8 \pm 6.1$ years) and 17 women with twin pregnancies (mean age, $25.2 \pm 3.8$ years; $P=.01$ ). The gestational ages at the time of evaluation were $31.7 \pm 1.5$ and $32.7 \pm 2.3$ weeks, respectively $(P=.09)$. The prepregnancy BMIs were $22.4 \pm 3.6$ and $25.2 \pm 5.2 \mathrm{~kg} / \mathrm{m}^{2}(P=.06)$. The BMIs at the time of evaluation were $26.3 \pm 3.5$ and $30.0 \pm 5.1 \mathrm{~kg} / \mathrm{m}^{2}(P=.01)$. 
The mean sagittal diameters at rest, during the Valsalva maneuver, and during contraction were $5.3 \pm 0.7,5.7 \pm 0.6$, and $4.5 \pm 0.6 \mathrm{~cm}$, respectively, in women with singleton pregnancies and $5.3 \pm 0.6,5.8 \pm 0.9$, and $4.6 \pm 0.5 \mathrm{~cm}$ in women with twin pregnancies (Table 1). The mean coronal diameters at rest, during Valsalva, and during contraction were $3.8 \pm 0.4,4.1 \pm 0.4$, and $3.6 \pm 0.4 \mathrm{~cm}$ in women with singleton pregnancies and $4.3 \pm 0.3,4.3 \pm 0.5$, and $3.8 \pm 0.3 \mathrm{~cm}$ in women with twin pregnancies. Statistical significance was observed at rest and during contraction $(P<.01 ; P=.04)$. The mean levator hiatal areas at rest, during Valsalva, and during contraction were $14.6 \pm 2.3,16.9 \pm 2.5$, and $11.7 \pm 2.4 \mathrm{~cm}^{2}$ in women with singleton pregnancies and $16.0 \pm 2.9,18.6 \pm 5.1$, and $12.6 \pm 2.3 \mathrm{~cm}^{2}$ in women with twin pregnancies.

\section{Discussion}

Pregnancy causes biomechanical, neurologic, neuromuscular, and hormonal changes in the pelvic floor. These changes have previously been demonstrated as changes in the levator hiatal area by comparing nonpregnant women with pregnant women in their third trimester. ${ }^{11}$ Various factors can explain these changes; eg, the additional weight and greater BMI during pregnancy results in increased loading of the pelvic floor, and smooth muscle relaxation increases because of the effect of hormones such as progesterone.

In twin pregnancies, both muscle relaxation and mechanical loading are present to a greater degree than in singleton pregnancies. However, to our knowledge, no studies have evaluated the changes in the pelvic floor of women with twin pregnancies using transperineal 3D

Figure 1. Transperineal 3D sonography of the pelvic floor. A convex transducer was used in the multiplanar mode. The images show the sagittal plane (A), coronal plane (B), axial plane (C), and, in the rendered mode (3D), the pubic symphysis, urethra, vagina, and rectum.

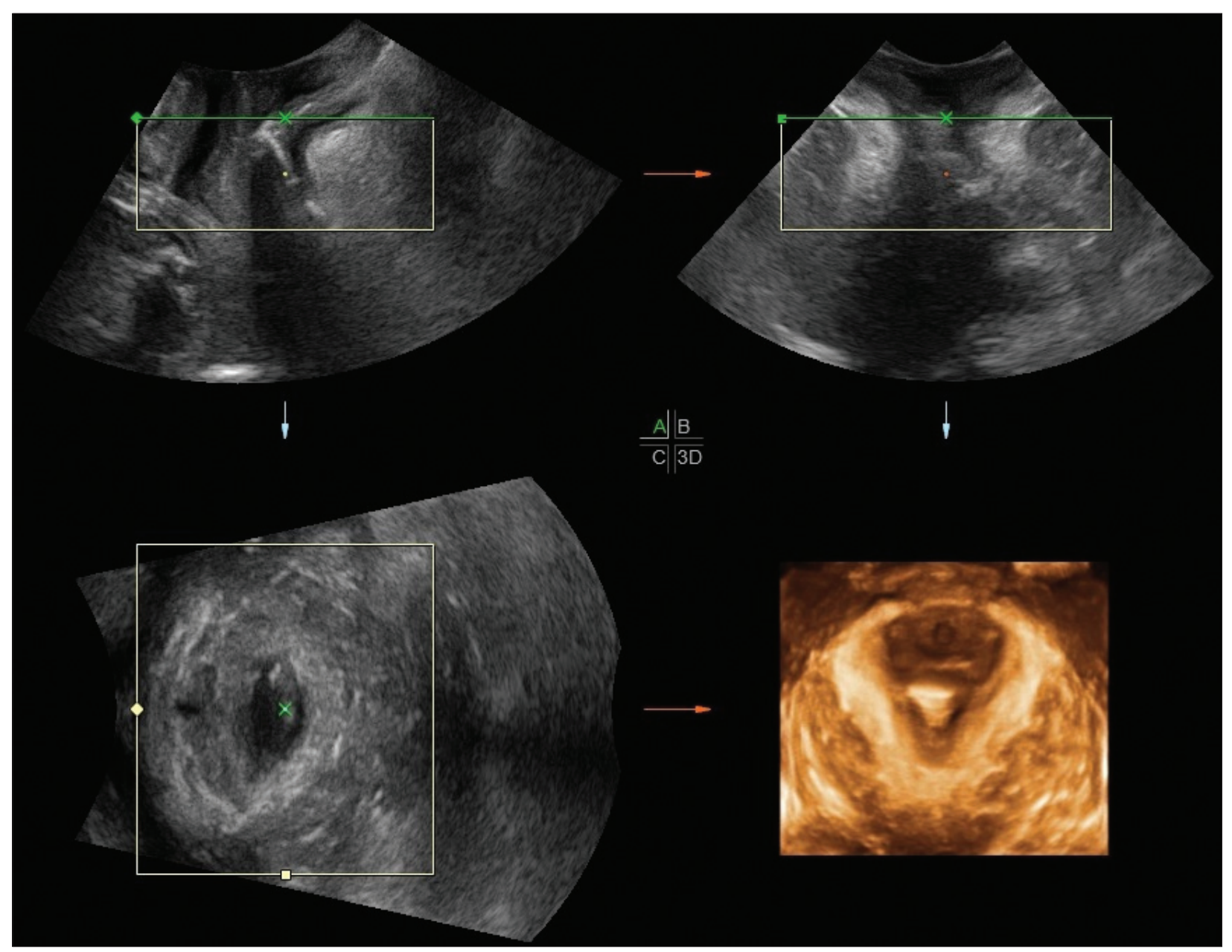


sonography. Some authors have correlated twin pregnancy and the type of delivery with urinary incontinence, stress urinary incontinence, urge urinary incontinence, fecal incontinence, and gas incontinence using questionnaires alone ${ }^{6-8}$; however, none have physically evaluated the pelvic floor or made comparisons with singleton pregnancies.

Regarding measurements of the levator hiatal area at rest, we established a value of $14.6 \mathrm{~cm}^{2}$ in women with singleton pregnancies. This value was similar to those of several other studies: $14.13,{ }^{12} 14.61,{ }^{13} 14.9,{ }^{14} 15.23,{ }^{15}$ $15.2{ }^{16}$ and $15.10^{11} \mathrm{~cm}^{2}$; however, it differed from the 11.81 $\mathrm{cm}^{2}$ reported by Lanzarone and Dietz. ${ }^{17}$ In addition, we measured the sagittal diameters in women with singleton pregnancies during rest, with results similar to those in the existing literature: a mean value of $5.3 \mathrm{~cm}$ in our study compared to the $5.13 \mathrm{~cm}$ obtained by Lanzarone and Dietz ${ }^{17}$ and the $5.51 \mathrm{~cm}$ obtained by Svabík et al. ${ }^{12}$ The coronal diameter at rest among women with singleton pregnancy was $3.8 \mathrm{~cm}$, which was similar to the 3.65 and $3.82 \mathrm{~cm}$ obtained by Lanzarone and Dietz ${ }^{17}$ and Svabík et al, ${ }^{12}$ respectively. When comparing our results with the literature on twin pregnancy, our measurements were generally higher, although not all differences reached significance.

The biometric measurement values for the levator hiatus of pregnant women are larger than those of nonpregnant women because the hormones produced during pregnancy affect the biochemical compositions of the solid matrix and hydration phases that constitute the pelvic floor tissues. Remodeling mechanisms alter the organization, orientation, and diameter of collagen fibers and recruit collagen fibrils for reinforcement of each tissue type. These effects substantially affect the viscoelastic properties of the vaginal wall, pubovisceral muscle, and perineal body over both short and long terms. In turn, these properties will deter-

Figure 2. Measurement of the levator hiatal area by transperineal 3D sonography.

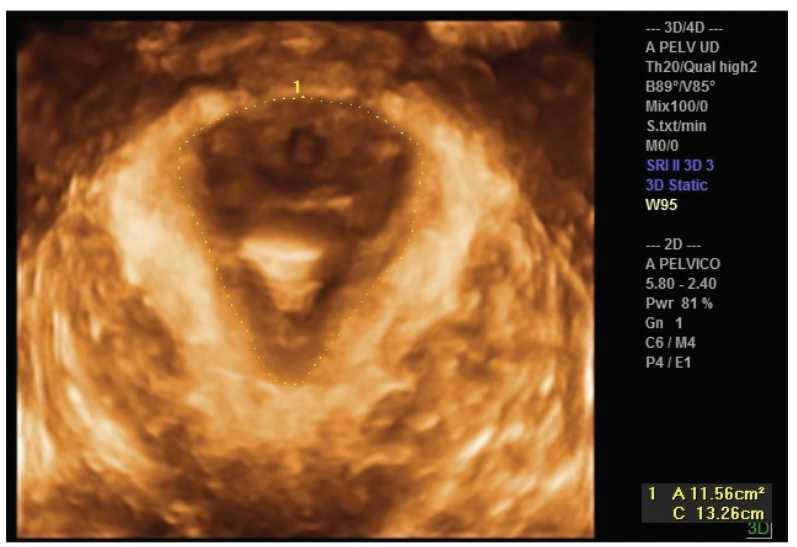

mine the distensibility during the expulsion phase of vaginal delivery and the resistance presented by these structures. ${ }^{18}$

During the second stage of labor, perineal distensibility is important to prevent birth trauma secondary to the high pressures imposed by the fetal head on the muscles of the pelvic floor. ${ }^{18}$ However, in twin pregnancies, this distensibility will probably be less because the individual fetal weights are typically lower than in singleton pregnancies. Studies using 3D sonography and magnetic resonance imaging have demonstrated that the puborectalis muscle needs to stretch substantially during vaginal delivery. ${ }^{19,20}$ Thus, greater perineal distensibility is important among pregnant women favoring vaginal delivery, although it may be less important in twin pregnancies.

In this study, the levator hiatal areas were greater among women with twin pregnancies (mean, $16.0 \mathrm{~cm}^{2}$ ) than among women with singleton pregnancies $\left(14.6 \mathrm{~cm}^{2}\right)$. Moreover, the levator hiatal area at rest tended to be greater among women with twin pregnancies $(P=.08)$. Our results suggest that the hormonal and mechanical conditions specific to twin pregnancy may affect the anatomy and function of the levator ani muscle.

The hiatal areas at rest and during Valsalva have been associated with signs and symptoms of pelvic organ prolapse, both in symptomatic and asymptomatic women., ${ }^{9,21}$ Thus, perineal musculature measurement is important for analysis. Measuring the distensibility of the levator hiatus is one of the most basic approaches for determining the biomechanical properties of this muscle. ${ }^{21}$

The main limitation of this study was the small sample size, likely resulting in a type II error for some measurements. The small sample size also prevented adequate control for confounders such as BMI, gestational age, and ethnic origin. Furthermore, because this study was cross-sectional,

Figure 3. Measurement of the sagittal and coronal diameters of the levator hiatus by transperineal 3D sonography.

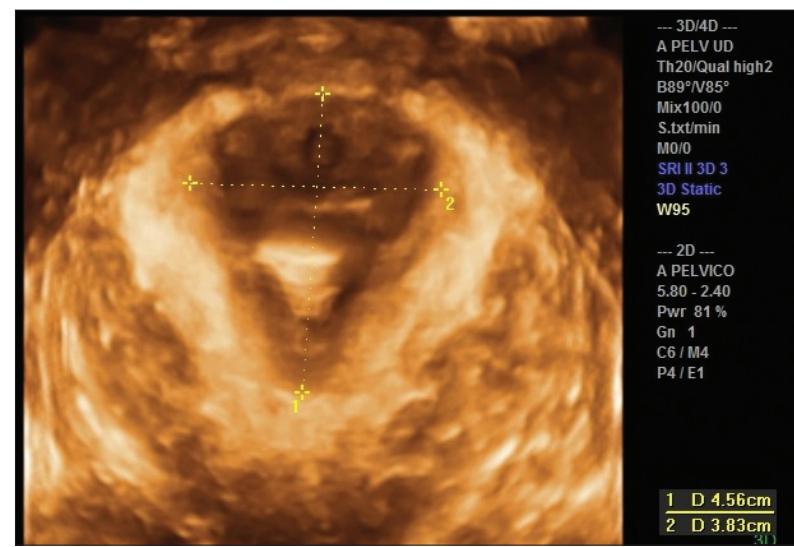


Table 1. Comparison of Levator Hiatus Measurements Between Singleton and Twin Pregnancies Evaluated by Transperineal 3D Sonography

\begin{tabular}{|c|c|c|c|c|c|c|c|}
\hline \multirow[b]{2}{*}{ Measurement } & \multirow[b]{2}{*}{$\mathbf{n}$} & \multicolumn{2}{|l|}{ Rest } & \multicolumn{2}{|c|}{ Valsalva } & \multicolumn{2}{|c|}{ Contraction } \\
\hline & & Mean \pm SD & $P$ & Mean \pm SD & $P$ & Mean \pm SD & $P$ \\
\hline \multicolumn{8}{|c|}{ Sagittal diameter, cm } \\
\hline Singleton & 23 & $5.3 \pm 0.7$ & \multirow{2}{*}{$.88^{a}$} & $5.7 \pm 0.6$ & \multirow{2}{*}{$.57^{a}$} & $4.5 \pm 0.6$ & \multirow{2}{*}{$.51^{a}$} \\
\hline Twin & 17 & $5.3 \pm 0.6$ & & $5.8 \pm 0.9$ & & $4.6 \pm 0.5$ & \\
\hline \multicolumn{8}{|c|}{ Coronal diameter, cm } \\
\hline Singleton & 23 & $3.8 \pm 0.4$ & \multirow{2}{*}{$<.01^{a}$} & $4.1 \pm 0.4$ & \multirow{2}{*}{$.31^{a}$} & $3.6 \pm 0.4$ & \multirow{2}{*}{$.04^{a}$} \\
\hline Twin & 17 & $4.3 \pm 0.4$ & & $4.3 \pm 0.5$ & & $3.8 \pm 0.3$ & \\
\hline \multicolumn{8}{|l|}{ Hiatal area, $\mathrm{cm}^{2}$} \\
\hline Singleton & 23 & $14.6 \pm 2.3$ & \multirow{2}{*}{$.08^{a}$} & $16.9 \pm 2.5$ & \multirow{2}{*}{$.22^{a}$} & $11.7 \pm 2.4$ & \multirow{2}{*}{$.23^{\mathrm{a}}$} \\
\hline Twin & 17 & $16.0 \pm 2.9$ & & $18.6 \pm 5.1$ & & $12.6 \pm 2.3$ & \\
\hline
\end{tabular}

Each patient was observed only once.

aUnpaired Student $t$ test.

we did not obtain postnatal data. There was also no postnatal follow-up; as a result, we could not determine whether pelvic floor changes were reversible (eg, hormonal/ mechanical factors) or irreversible (eg, those resulting from levator ani trauma). Larger studies incorporating postnatal follow-up are required to confirm these results.

Despite these weaknesses, we believe that this study is an important contribution to the literature because it provides physical data on the effect of twin pregnancy on the pelvic floor. We have demonstrated increased coronal diameters at rest and during contraction, thereby suggesting that pelvic support undergoes greater changes during twin pregnancy.

\section{References}

1. Chen B, Wen Y, Yu X, Polan ML. Elastin metabolism in pelvic tissues: is it modulated by reproductive hormones? Am J Obstet Gynecol 2005; 192:1605-1613.

2. South MM, Stinnett SS, Sanders DB, Weidner AC. Levator ani denervation and reinnervation 6 months after childbirth. Am JObstet Gynecol 2009; 200:519.e1-519.e7.

3. Wijma J, Potters AE, de Wof BT, Tinga DJ, Aarnoudse JG. Anatomical and functional changes in the lower urinary tract following spontaneous vaginal delivery. BJOG 2003; 110:658-663.

4. O'Boyle AL, O'Boyle JD, Ricks RE, Patience TH, Calhoun B, Davis G. The natural history of pelvic organ support during pregnancy. Int Urogynecol J2003; 14:46-49.

5. Netto HC,Moreira de Sá RA (eds). Obstetrícia Básica. 2nd ed. São Paulo, Brazil: Atheneu; 2007.

6. Goldberg RP, Kwon C, Gandhi S, Atkuru LV, Sorensen M, Sand PK. Urinary incontinence among mothers of multiples: the protective effect of cesarean delivery. Am J Obstet Gynecol 2003; 188:1447-1450.

7. Legendre G, TasselJ, Salomon LJ, Fauconnier A, Bader G. Impact of twin gestation on the risk of postpartum stress incontinence [in French]. Gynecol Obstet Fertil 2010; 38:238-243.
8. Cuerva González MJ, López Carpintero N, de la Calle Fernández MM, Usandizaga R, González A. Postpartum urinary and fecal incontinence in gemelar pregnancy according to route and mode of delivery [in Spanish]. Ginecol Obstet Mex 2011; 79:540-546.

9. Dietz HP, Shek C, Clarke B. Biometry of the pubovisceral muscle and levator hiatus by three-dimensional pelvic floor ultrasound. Ultrasound Obstet Gynecol 2005; 25:580-585.

10. Cochran W. Sampling Techniques. 2nd ed. Boston, MA: John Wiley \& Sons; 1985.

11. Shek KL, Kruger J, Dietz HP. The effect of pregnancy on hiatal dimensions and urethral mobility: an observational study. Int Urogynecol J2012; 23:1561-1567.

12. Svabík K, Shek KL, Dietz HP. How much does the levator hiatus have to stretch during childbirth? BJOG 2009; 116:1657-1662.

13. Shek KL, Dietz HP. The effect of childbirth on hiatal dimensions. Obstet Gynecol 2009; 113:1272-1278.

14. Shek $\mathrm{KL}$, Dietz HP. Intrapartum risk factors of levator trauma. BJOG 2010; 117:1485-1492.

15. Shek KL, Dietz HP. Can levator avulsion be predicted antenatally? Am J Obstet Gynecol 2010; 206:586.e1-586.e6.

16. Wu QK, Mao XY, Luo LM, Ying T, Li Q, Teng YC. Characteristics of pelvic diaphragm hiatus in pregnant women with stress urinary incontinence detected by transperineal three-dimensional ultrasound [in Chinese]. Zhonghua Fu Chan Ke Za Zhi2010; 45:326-330.

17. Lanzarone V, Dietz HP. Three-dimensional ultrasound imaging of the levator hiatus in late pregnancy and associations with delivery outcomes. Aust NZJ Obstet Gynaecol 2007; 47:176-180.

18. Ashton-MillerJA,DelanceyJO. On the biomechanics of vaginal birth and common sequelae. Annu Rev Biomed Eng 2009; 11:163-176.

19. Dietz HP. Levator function before and after childbirth. Aust NZ J Obstet Gynaecol 2004; 44:19-23.

20. Dietz HP, Gillespie AV, Phadke P. Avulsion of pubovisceral muscle associated with large vaginal tear after normal vaginal delivery at term. Aust NZJ Obstet Gynaecol 2007; 47:341-344.

21. Dietz HP, Shek C, De Leon J, Steensma AB. Ballooning of the levator hiatus. Ultrasound Obstet Gynecol 2008; 31:676-680. 